Punto de vista

\title{
Análisis de los «casi errores». Un nuevo enfoque en el estudio de los acontecimientos adversos
}

\author{
I. GUTIÉRREZ CÍAa , B. OBÓN AZUARAa Y C. AIBAR REMÓN ${ }^{b}$

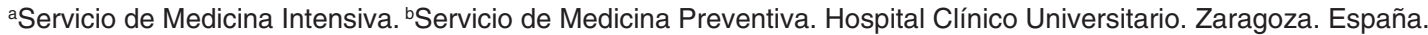

\begin{abstract}
Se define «casi error» como aquella situación que, de no haber sido evitada, hubiera podido provocar daño al paciente. El análisis de los casi errores puede resultar una eficaz herramienta en el estudio de los acontecimientos adversos. El registro de los casi errores ofrece numerosas ventajas sobre el estudio de acontecimientos adversos, ya que los casi errores ocurren con mayor frecuencia que los acontecimientos adversos y además, al carecer de consecuencias, se minimiza el temor que los profesionales implicados pudieran tener a declararlos. Sin embargo, hasta este momento es un método que se ha utilizado con poca frecuencia. Presentamos dos casos clínicos que ayudan a comprender la utilidad del registro y análisis de los casi errores.
\end{abstract}

PALABRAS CLAVE: casi error, acontecimiento adverso, Cuidados Intensivos.

\section{A NEAR-MISS EVENT. A NEW APPROACH IN THE STUDY OF ADVERSE EVENTS}

A near-miss event is defined as an event that could have resulted in an injury, fatality, or property damage if it had not been prevented. Analysis of near-miss events could be an efficient method in the study of adverse events. Reporting of near-misses has many benefits in the study of adverse events since near-misses occur more frequently than adverse events. In addition, as they have no consequences, fear that the profes-

Correspondencia: Dra. I. Gutiérrez Cía.

Servicio de Medicina Intensiva.

Hospital Clínico Universitario.

Avda. San Juan Bosco, 15, $1^{\mathrm{a}}$ planta.

50009 Zaragoza. España.

Correo electrónico: igutierrcia@hotmail.com

Manuscrito aceptado el 1-VI-2007. sionals involved would have to report them is less. However, up to now, this method has been slow to develop. We present two clinical cases that help to understand the usefulness of the near-miss reporting system.

KEY WORDS: near-miss, adverse event, Intensive Care.

\section{INTRODUCCIÓN}

La preocupación por «no hacer daño» ha estado siempre presente en el ánimo de los profesionales sanitarios. Cada decisión que tomamos, cada tratamiento que pautamos, cada técnica que realizamos con el objetivo de mejorar la salud del paciente conlleva la posibilidad de generar efectos secundarios, complicaciones, acontecimientos adversos (AA) e incluso errores. Es decir, el riesgo asistencial y la incertidumbre en la toma de decisiones son consustanciales a la práctica clínica.

\section{ACONTECIMIENTOS ADVERSOS Y CASI ERRORES}

La Sociedad Española de Medicina Intensiva definió AA como aquel daño no intencionado causado durante o a consecuencia de la atención sanitaria y no relacionado con la evolución o posibles complicaciones de la enfermedad de base del paciente ${ }^{1}$. Complementando a este término encontramos otro, no muy bien definido, pero de indudable utilidad para el aprendizaje, denominado «casi error» (CE) (tabla 1$)^{2-6}$. Los CE son situaciones que, de no haber sido evitadas, hubieran podido provocar daño al paciente. El estudio de los CE ha demostrado su utilidad en otros ámbitos como la aviación o la energía nuclear, donde han sido analizados tan exhaustivamente como los $\mathrm{AA}^{6}$. 
TABLA 1. Definiciones de «casi error»

\begin{tabular}{|r|l|}
\hline Autor & Definición \\
\hline Van der Schaff $^{3}$ & $\begin{array}{l}\text { Caso en el que el accidente ha sido evitado por poco } \\
\text { Cualquier situación en la que una sucesión continuada de efectos fue detenida evitando la } \\
\text { aparición de potenciales consecuencias }\end{array}$ \\
March $^{4}$ & $\begin{array}{l}\text { Hecho que casi ocurrió } \\
\text { Ives }^{5}\end{array}$ \\
$\begin{array}{l}\text { Suceso que en otras circunstancias podría haber tenido graves consecuencias } \\
\text { Acontecimiento peligroso que no ha producido daños personales, pero sí materiales, y que } \\
\text { sirve de aviso de posibles sucesos }\end{array}$ \\
\hline
\end{tabular}

Los métodos empleados con más frecuencia para el estudio de los AA son el análisis de quejas y demandas, el estudio de autopsias, la revisión de bases de datos administrativos clínicas, la revisión de historias clínicas, la observación directa y los programas de declaración voluntaria de $\mathrm{AA}^{7}$. Este último es uno de los métodos más utilizados junto con la observación directa para la detección de los $\mathrm{CE}$, ya que son situaciones de riesgo que podrían dar lugar a AA que en realidad no llegan a ocurrir, por lo que nada queda reflejado en la historia clínica, no dan lugar a demandas, no se traducen en lesiones visibles en una autopsia, ni figuran en ninguna base de datos.

La observación directa de la atención al paciente, aunque es un procedimiento potencialmente preciso, es costosa y presenta problemas de entrenamiento de observadores. Estas razones convierten a los programas de declaración voluntaria en el método más apropiado para la detección de los $\mathrm{CE}$.

Los sistemas de detección de AA mediante programas de declaración voluntaria tienen como objetivo principal aprender de la experiencia ${ }^{8}$. El problema fundamental de los sistemas de declaración voluntaria es que no identifican el número real de AA, como se ha comprobado al comparar este método con otros ${ }^{9-11}$. No siempre se declaran los $\mathrm{AA}^{12}$. Nos encontramos en una sociedad que espera resultados y exige responsabilidades al sistema sanitario y a sus profesionales, lo que provoca con frecuencia reacciones de miedo y ocultación ante los AA. Esto, unido a la falta de confianza en la utilidad de la notificación ${ }^{13}$, provoca rechazo a los programas de detección y análisis de AA. Para hacer de los sistemas de declaración voluntaria las herramientas más útiles en la mejora de la seguridad del paciente deben asegurar el anonimato y ser útiles para establecer estrategias de prevención.

Desde este punto de vista, el estudio de los CE aporta claras ventajas sobre el de los $\mathrm{AA}^{14}$. Por una parte, son situaciones que permiten un análisis similar, y presentan menor recelo a su notificación ya que no han tenido consecuencias ${ }^{15}$. Por otra, suceden con mucha mayor frecuencia ${ }^{14,16}$. Así, por ejemplo, entre los años 2003 y 2005 se estudiaron los CE relacionados con las transfusiones de hemoderivados en Irlanda. Los AA relacionados con transfusiones fueron muy poco habituales, pero los CE ocurrieron con mucha frecuencia, lo que les proporcionó una importante fuente de datos para detectar fallos en el sistema que de otra forma hubieran pasado desapercibidos ${ }^{17}$.

Del correcto análisis de un CE podemos obtener la misma información en cuanto a posibilidades de pre- vención que de un auténtico AA. En ambos casos aprendemos de la experiencia, pero los CE nos ofrecen una «enseñanza gratuita» ${ }^{18}$ que no debemos despreciar.

Se exponen a continuación dos casos que ayudan a comprender la importancia del estudio de los CE.

\section{DESCRIPCIÓN DE LOS CASOS}

\section{Caso 1}

Se trata de un paciente de 55 años, sin enfermedades previas, que sufre traumatismo torácico y pélvico producido tras una caída desde 5 metros de altura. En el lugar del accidente presenta GCS 15 e insuficiencia respiratoria con hipoventilación del pulmón izquierdo, por lo que se implanta un pleurecath. Es ingresado en la Unidad de Cuidados Intensivos (UCI) donde se practica radiografía de tórax en la que se aprecian fracturas costales izquierdas múltiples, contusión pulmonar bilateral y existencia de un catéter que parece llegar hasta pericardio. En la tomografía axial computarizada (TAC) pulmonar con contraste practicada de modo urgente, además de lo mencionado, se objetiva que dicho tubo atraviesa el parénquima pulmonar y se encuentra adyacente al margen del cayado aórtico (fig. 1). Tras retirada de este drenaje el paciente es dado de alta tras 72 horas de permanencia en UCI.

\section{Caso 2}

Se trata de un paciente ingresado en UCI para control posquirúgico inmediato tras haber sido intervenido de una resección de colon transverso motivada por un proceso neoplásico. A su ingreso se pautó el tratamiento analgésico habitual en nuestro servicio, consistente en una perfusión continua durante 24 horas de una combinación de metamizol, tramadol y metoclopramida. La figura 2 muestra la redacción del tratamiento, en la que se da por supuesto que se comprende la vía de administración, sin embargo, en las prescripciones no figura explícitamente. La enfermera responsable del paciente se disponía a proporcionar la analgesia mencionada por vía oral cuando fue sorprendida por la auxiliar de clínica, quien le informó de que el tratamiento se administraba habitualmente por vía intravenosa.

\section{ANÁLISIS DE LOS CASOS}

Los casos expuestos demuestran la utilidad del estudio de los $\mathrm{CE}$ en dos vertientes muy diferentes. Por 


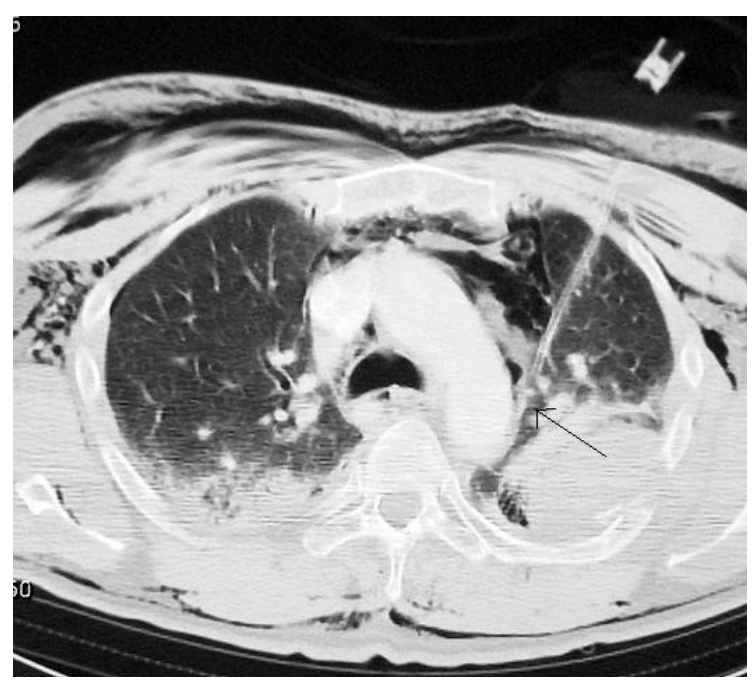

Figura 1. Tomografía axial computarizada torácica con contraste: tubo adyacente al margen del cayado aórtico (flecha). Contusión pulmonar. Hemotórax izquierdo loculado.

una parte, el caso 1 nos ayuda en el estudio de una situación muy infrecuente. En el caso 2 nos encontramos ante un CE de medicación. Los errores de medicación, aunque frecuentes, no siempre son declarados especialmente cuando han provocado un daño al paciente, ya que podrían traducirse en una pérdida de prestigio. El caso que presentamos no tuvo consecuencia pues se trataba de un $\mathrm{CE}$, lo que facilitó su análisis ya que se minimizó la sensación de miedo a la responsabilidad.

Ambas situaciones fueron analizadas en las sesiones rutinarias que se llevan a cabo en nuestro servicio.

\section{Caso 1}

El caso 1 resultó de gran utilidad en el estudio de las complicaciones y AA derivados de la implantación de drenajes pleurales. La TAC nos muestra un AA evidente: la colocación intraparenquimatosa del drenaje. Esta es una situación que se observa con relativa frecuencia y que puede ser causa de complicaciones tales como hemorragia o fuga aérea, pero al observar atentamente la imagen nos dimos cuenta de que el tubo «casi» llegaba a la aorta y potencialmente podría haber causado una lesión vascular grave. Este tipo de lesiones son muy infrecuentes y altamente improbables, encontrando en la literatura escasas comunicaciones referentes a lesiones vasculares secundarias a una incorrecta inserción de un tubo torácico ${ }^{19,20}$. Sin embargo, aunque infrecuentes, son situaciones que revisten una extrema gravedad y que ponen en peligro la vida del paciente.

El análisis de este caso fue especialmente interesante para la formación de los residentes de nuestro servicio, quienes comprendieron que el empleo de técnicas aun sencillas como la implantación de un drenaje torácico mediante dispositivos como pleurecath debe hacernos valorar, además de su indicación,

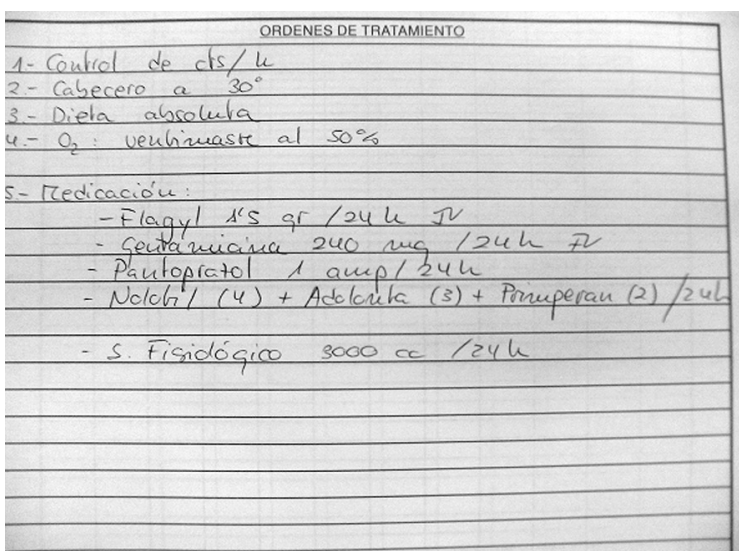

Figura 2. Órdenes de tratamiento en la gráfica del paciente.

la práctica de los mismos por personal entrenado en aras de evitar la presentación de AA, que, como en el presente caso, podría haber precisado una intervención quirúrgica urgente de la aorta, o bien haber comportado el fallecimiento del paciente en el lugar de los hechos.

\section{Caso 2}

Los errores en la vía de administración de los fármacos prescritos son frecuentes e incluso han motivado diversos estudios acerca de los AA. El análisis del caso expuesto nos demostró la concurrencia de dos factores: en primer lugar, la incompleta redacción del tratamiento prescrito, confiando en que se trataba de una perfusión muy habitual en nuestro servicio, conocida por todo el personal de enfermería. Sin embargo, no se tuvo en cuenta la posible inexperiencia de la enfermera responsable del paciente, como factor contribuyente a la aparición de AA. El hecho ocurrió durante período vacacional, por lo que parte del personal de enfermería se encontraba trabajando por primera vez en la UCI.

Este hecho se analizó en la sesión clínica de la mañana siguiente con la colaboración de la supervisora de enfermería y del facultativo implicado, con el objetivo de intentar que no volviera a suceder. Nos permitió incidir en la necesidad de contar con personal adecuadamente formado $\mathrm{y}$, siempre que fuera posible, con experiencia en el cuidado del enfermo crítico. Pero además, al personal que se incorpora por primera vez a un servicio se le debe proporcionar formación acerca de cuál debe ser su actuación en el mismo. Por esto, nuestro servicio cuenta con una «guía de acogida», pero este hecho expuesto nos hizo ver la posibilidad de introducir mejoras en la misma, incluyendo información acerca de los protocolos más habituales.

Por otra parte, nunca debe dejarse de insistir en la necesidad de detallar al máximo las prescripciones; incluso las que se utilizan más habitualmente en la 
UCI deben escribirse correctamente, haciendo constar siempre la dosis, la dilución y la vía de administración. Probablemente un sistema informatizado de las prescripciones de tratamiento constituiría un método de prevención adecuado para evitar este tipo de acontecimientos $^{21}$.

Así, del análisis de estas dos situaciones que «afortunadamente no ocurrieron», no solo hemos obtenido una enseñanza práctica, sino que hemos detectado importantes oportunidades de mejora.

\section{CONSIDERACIONES FINALES}

Las UCI son espacios especialmente favorables para la implantación de programas de detección y análisis de los CE. La constante presencia de los especialistas en la unidad y una ratio personal de enfermería/paciente muy superior a la que podemos encontrar en una planta de hospitalización son hechos que facilitan su detección. La adecuada dotación de facultativos y de personal de enfermería contribuye además a que la historia clínica en UCI sea exhaustiva y cuente con un registro horario de constantes vitales, tratamientos y técnicas realizadas, lo que se traduce en una mayor facilidad de análisis de cada CE. Además, los indicadores de calidad del enfermo crítico, redactados recientemente, están, en muchos casos, relacionados con la seguridad del enfermo ${ }^{22}$, lo que facilita el reconocimiento de los AA y CE, el análisis de las causas raíz y la identificación de muchos de los errores latentes.

La estructura del trabajo en la UCI se apoya en sesiones diarias en las que se valora el diagnóstico, evolución y tratamiento de nuestros pacientes. Creemos que además pueden ser un marco adecuado para difundir una cultura de seguridad, comentando y analizando brevemente este tipo de situaciones, incorporando la detección de CE y AA a nuestra labor habitual. Esta rutina nos permitirá también seleccionar casos para sesiones específicas.

Por último, creemos necesario destacar que, como ilustra el caso 1, con frecuencia en la UCI detectamos CE y AA ocurridos en los servicios que nos preceden en la escala asistencial. Es necesario colaborar en la formación de los profesionales de otros servicios, cuya labor asistencial puede beneficiarse de técnicas otrora exclusivas de las UCI. Y en este sentido nos planteamos en el momento actual la realización de sesiones conjuntas en las que el análisis de estos acontecimientos puede convertirse en una eficaz herramienta de aprendizaje.

Los CE no ocasionan deterioro de la salud del paciente ni suelen prolongar su estancia. Su registro y análisis supone una importante fuente de información y una oportunidad para detectar fallos en el sistema y aprender de nuestros errores antes de que produzcan fatales consecuencias. Hasta el momento existen pocos datos sobre la incidencia de los CE, por ello deberían incluirse en todos los registros voluntarios de AA.

\section{Declaración de conflicto de intereses}

Los autores han declarado no tener ningún conflicto de intereses.

\section{BIBLIOGRAFÍA}

1. Grupo de trabajo de organización, planificación y gestión de la SEMICYUC. Prevalencia de incidentes y acontecimientos adversos en los servicios de Medicina Intensiva. Descripción del proyecto (consultado el 21-05-07). Disponible en: www.semicyuc.org

2. Vincent C, Ennis M, Audley RJ. Medical accidents. Oxford: Oxford University Press; 1993.

3. Van der Schaff TW. Development of a near miss management system at a chemical process plant. En: Van der Schaff TW, Hale AR, Lucas DA, editors. Near miss reporting as a safety tool. Oxford: Butterworth-Heinemann; 1991.

4. March JG, Sproull LS, Tamuz M. Learning from samples of one or fewer. 1991. Qual Saf Health Care. 2003;12:465-71.

5. Ives G. Near miss reporting pitfalls for nuclear plants. En: Van der Shaff TW, Lucas DA, Hale AR, editors. Near miss reporting as a safety tool. Oxford: Butterworth-Heineman; 1991.

6. US Nuclear Regulatory Commission. Reporting safety concerns to the NRC. Washington, DC: US Nuclear Regulatory Commission; 1998.

7. Thomas EJ, Petersen LA. Measuring errors and adverse events in health care. J Gen Intern Med. 2003;18:61-7.

8. Leape LL. Reporting of adverse events. N Engl J Med. 2002;347:1633-8

9. Stanhope N, Crowley-Murphy M, Vincent C, O'Connor AM, Taylor-Adams SE. An evaluation of adverse incident reporting. J Eval Clin Pract. 1999;5:5-12.

10. Capuzzo M, Nawfal I, Campi M, Valpondi V, Verri M, Alvisi R. Reporting of unintending events in an intensive care unit: comparison between staff and observer. BMC Emerg Med. 2005; 5:1-79.

11. Rothschild JM, Keohane CA, Cook EF, Orav EJ, Burdick E, Thompson S, et al. A controlled trial of smart infusion pumps to improve medication safety in critically ill patients. Crit Care Med. 2005;33:533-40

12. Vincent C, Stanhope N, Crowley-Murphy M. Reason for not reporting adverse incidents: an empirical study. J Eval Clin Pract. 1999;5:13-21.

13. Leape LL. Why should we report adverse incidents? J Eval Clin Pract. 1999;5:1-4.

14. Barach $P$, Small SD. Reporting and preventing medical mishaps: lesson and safety in clinical medicine. BMJ. 2000;320:759-63.

15. Aranaz JM, Aibar C, Galán A, Limón R, Requena J, Álvarez $\mathrm{EE}$, et al. La asistencia sanitaria como factor de riesgo: los efectos adversos ligados a la práctica clínica. Gac Sanit. 2006;20 Supl 1: 41-7.

16. Van der Schaff TW. Near miss analysis. En: Aspen P, Corrigan J, Erickson S, editors. Patient safety: achieving a new standard for care. Washington DC: National Academy Press; 2004:226-46.

17. Lundy D, Laspina S, Kaplan H, Fastman BR, Lawlor E. Seven hundred and fifty-nine (759) chances to learn: a 3-year pilot project to analyse transfusion-related near-miss events in the Republic of Ireland. Vox Sang. 2007;92:233-41.

18. Spencer FC. Human error in hospitals and industrial accidents: current concepts. J Am Coll Surg. 2000;191:410-8.

19. Valette H, Giustozzi A. Another complication of thoracic drainage: perforation-intubation of the subclavian vein. J Chir (Paris). 1996;133:91-2.

20. Kerger H, Blaettner T, Froehlich C, Ernst J, Friestch T, Isselhorst $\mathrm{C}$, et al. Perforation of the left atrium by a chest tube in a patient with cardiomegaly: management of a rare, but life-threatening complication. Resuscitation. 2007:74:178-82.

21. Bates DW, Leape LL, Cullen DJ, Laird N, Petersen LA, Teich JM, et al. Effect of computerized physician order entry and a team intervention on prevention of serious medication errors. JAMA. 1998;280:1311-6.

22. Martín MC, Ruiz J. Acontecimientos adversos en Medicina Intensiva. Gestionando el riesgo. Med Intensiva. 2006;30:284-92. 